\title{
Piles in chalk under axial loading
}

\author{
Mirna Doghman ${ }^{1, *}$, Hussein Mroueh ${ }^{1}$, and Sébastien Burlon ${ }^{2}$ \\ ${ }^{1}$ LGCgE, University of Lille, 59655 Villeneuve d'Ascq, France \\ ${ }^{2}$ Terrasol, 75012 Paris, France
}

\begin{abstract}
Chalk exhibits a viscous behavior depending on time and it presents a noticeable creep under constant load. Consequently, piles founded in chalk media may have their design greatly affected. However, the different standards of the design of deep foundations don't take into account this viscous behavior and its effect on the pile settlement in the long term. This paper deals with the case of piles under monotonic axial loads in chalk. Two methods of predicting pile settlement are developed: the transfer curves method t$\mathrm{z}$, taking into account the viscosity, and the macroelement technique.
\end{abstract}

\section{Introduction}

Chalk has been the subject of several studies during these last decades because of its very particular mechanical behavior, as a soft porous rock. Chalk has a nonlinear stress-strain relationship, combining reversible and irreversible deformations [1], with the particularity that deformation in the chalk do not occur only because of instantly applied stresses, but also because of time-dependent mechanisms.

Rupture of structures in this soft rock can occur due to creep deformations [2]. Several studies carried out on chalk in different laboratories, [1], [3], have shown that this material exhibits a notable creep under constant load. Nevertheless, current regulations such as [4] or [5] do not directly take into account viscous behavior of chalk and its effect on the relation load-settlement of the pile.

This paper aims to study the mechanical behavior of a pile in chalk, under axial loading. Two techniques of pile modelling under monotonic axial load are used and compared in this paper to simulate the behavior of piles in the chalk: the transfer curves method and the macroelement technique. The transfer curves method implements a viscous t-z law based on the elasto-viscoplastic rheological model of Bingham. While considering the soil-structure interaction, the development of a macroelement consists in modeling the soil-pile set by a single element with one degree of freedom. The macroelement is developed according to an elasto-viscoplastic constitutive law including isotropic hardening.

\section{The transfer curves method (t-z)}

The transfer curve method, also called the t-z method was first described by [6]. It consists in modeling the pile as a structural element supported by discrete springs that represent the resistance of the soil along the shaft and the base of the pile. The pile is represented by an elastic beam discretized into a series of segments with properties that evolve with depth. Each element of the pile is associated with a transfer curve connecting the stress transmitted by this element to its displacement.

\subsection{Development of t-z viscous model}

Several experimental and theoretical load transfer curves, linear or not, exist in the literature. However, none of these models led to a presentation that combines the non-linear and viscous behavior of the soil. In the following, in order to take into account, the time-dependency behavior of the chalk, a viscous t-z model based on the elasto-viscoplastic rheological model of Bingham is presented.

Creep and viscosity effect are introduced in the $\mathrm{t}-\mathrm{z}$ method by modeling axial friction along the pile by the Bingham model (figure 1) formed of a spring in series with a set consisting of a dashpot and a sliding friction element in parallel.

\footnotetext{
*Corresponding author: mirna.doghman.etu@univ-lille.fr
} 


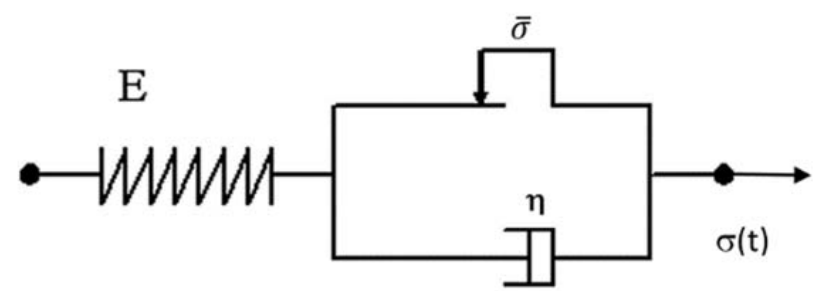

Fig. 1. Bingham model: $E$ stands for the Young's modulus, $\overline{\boldsymbol{\sigma}}$ for the plastic limit and $\eta$ for the viscosity

The modeling of the behavior of the soil based on this model is managed by the following formulas:

$$
\begin{gathered}
\sigma=\sigma_{1}=E * \varepsilon_{1} \\
\sigma=\sigma_{2}=\eta * \varepsilon_{2} \\
\dot{\varepsilon}=\dot{\varepsilon}_{1}+\dot{\varepsilon}_{2}
\end{gathered}
$$

Two cases may occur:

- If $\sigma<\bar{\sigma}$ :

The elastic domain governs the behavior of the soil characterized by equation 1 .

- If $\sigma>\bar{\sigma}$ :

The formulas 1 and 2 are injected in equation 3 to give the following differential equation:

$$
\frac{\sigma-\bar{\sigma}}{\eta}+\frac{\dot{\sigma}}{E}=\dot{\varepsilon}
$$

The solution of this equation is given by the following formula:

$$
\sigma=\sigma_{0} * e^{-\frac{E}{\eta} t}+\eta * \dot{\varepsilon}+\bar{\sigma}
$$

$\sigma_{0}$ denotes the stress in the previous phase.

The technique presented above is a typical resolution of the Bingham model. The difference with the non-linear $\mathrm{t}-\mathrm{z}$ laws is that the value of the plastic limit $\bar{\sigma}$ is no longer constant.

If the viscosity is different to zero, when the soil behavior exceeds the elastic limit the viscous behavior represented by the formula in the equation (5) generates the law of mobilization of the shaft friction along the pile. On the other hand, when the viscosity is zero, the axial friction $\tau$ follows the time-independent elastic-perfectly plastic behavior.

\subsection{Parametric study}

In this part, a parametric study is carried out to study the effect of the viscosity and the duration of application of the load on the behavior of the pile. The calculation was done firstly with a zero viscosity and secondly with a viscosity value of 100000 Pa.s.

For each value of the viscosity, loads are applied stepwise to a final value equal to the bearing capacity of the pile. Three periods of load application, 10,60 and $120 \mathrm{~min}$, are used to illustrate the behavior of the viscous soil, which depends essentially on time. Values of pile displacement are calculated every minute. The results are illustrated in the figure 2.

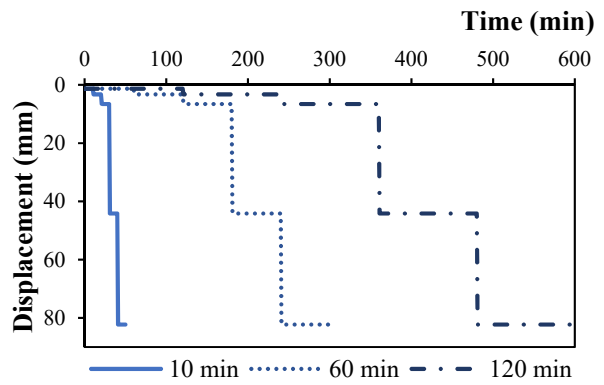

a)

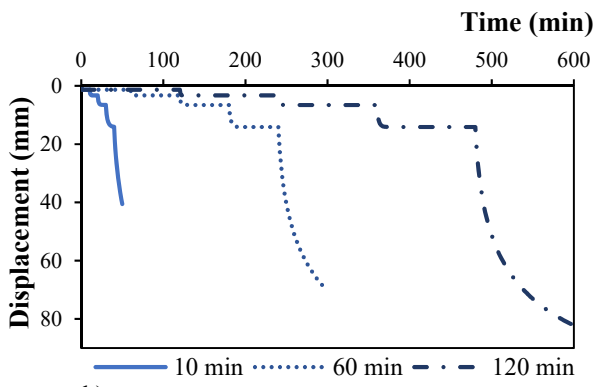

b)

Fig. 2. Displacement evolution with time: a) $\eta=0$, b) $\eta=100000$ Pa.s 
When the viscosity is zero (figure 2.a), the results found give a good representation of the behavior of the non-viscous soil where the settlements for each period of load application are the same. The displacement is constant in function of time. The absence of viscosity prevents soil creep and the pile does not experience delayed settlement over time.

However, the results in figure 2.b are different. Displacement under the same load evolves over time. Creep effect is well noticed and the compaction increases by increasing the duration of load application.

These results present the importance of taking into account the viscosity of the soil, the key aspect of soil creep, in the designing stage of piles. The evolution of pile settlement with time must be included in the norms to prevent the rupture in the long-term.

\subsection{Application to a case study}

Several in situ tests were carried out on piles in chalk in several cities of France within the LCPC (Laboratoire Centrale des Ponts et Chaussées). Among these tests, the one carried out at Fleury sur Andelle, [7], is chosen to do a comparison with calculation results from the developed viscous model.

Several full-scale static loading tests were carried out on a square pile, of $40 \mathrm{~cm}$ side and a length of $10.2 \mathrm{~m}$, at different times over almost one year in 1976 and 1977 with variable load application period. The chalk is considered the bearing layer, located below $3 \mathrm{~m}$ of clayed gravel. Numerical results, load-head displacement curves, are compared with the field data (figure 3).

The results show that the calculated results are basically conforming to the measured data, which indicates that the developed model showed its relevance on this site.
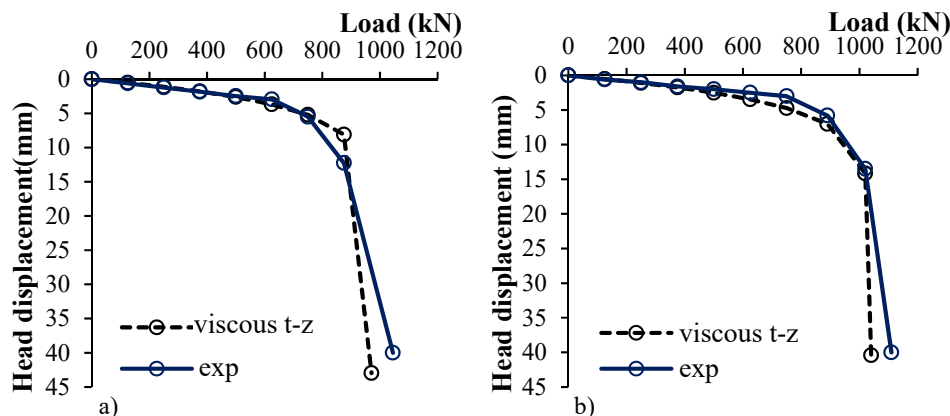

Fig. 3. Load-Head displacement curves: a) duration of load application 60min (28-4-1976), b) duration of load application 30min $(11-1-1977)$

\section{Development of a macroelement}

\subsection{Principle and formulation of the macroelement}

The macroelement allows a simple and direct analysis of soil-structure interaction problems that can occur at the foundation level. The behavior at the interface is thus reproduced in a global manner to save computing time. The concept of macroelement was initially introduced in the context of superficial foundations by [8] to simulate the behavior of a rigid foundation resting on a frictional soil.

The principle of a "macroelement" consists of replacing the soil-pile system by a single element with a correspondent number of degrees of freedom which is placed at the base of the superstructure and which aims to reproduce the nonlinear effects of the soil-structure interaction take place at the pile level.

The formulation of macroelement includes the implementation of an elasto-viscoplastic constitutive law and the flow rule that define the elastic and viscoplastic behavior of the soil-pile system. Thus, it is essential to determine the load surface and its evolution with viscoplastic displacement. In this present work, an isotropic hardening law is used.

The elastic behavior is defined according to equation (6):

$$
Q=k_{t} \cdot s^{e}
$$

$Q$ is the applied load, $k_{t}$ the stiffness of the spring and $s^{e}$ the elastic displacement.

The limit state $\mathrm{f}$ is defined by equation (7) and the flow rule is assumed to be associated.

$$
f: Q-Q_{c}=0
$$

$Q_{c}$ is the elastic limit evolving according to an isotropic hardening law, given by the following equations:

$$
Q_{c}=\left.Q_{c}\right|_{\dot{s}_{v 0}^{p}} *\left(\frac{\dot{s}_{v}^{p}}{\dot{s}_{v 0}^{p}}\right)^{\alpha}
$$




$$
\left.Q_{c}\right|_{\dot{s}_{v 0}^{p}}=Q_{c r}+\frac{a s_{v}^{p}}{b+s_{v}^{p}}
$$

The formula (8) is inspired from the expression proposed by [9] and then taken up by [10] to describe the evolution of the pre-consolidation pressure as a function of the rate of deformation. These expressions coming from the literature are written in terms of loads and displacements instead of stresses and deformations so that they are adapted to the current case.

$Q_{c r}$ is the creep load, $s_{v}^{p}$ is the viscoplastic displacement, $\dot{s}_{v}^{p}$ is the viscoplastic displacement velocity and $\dot{s}_{v 0}^{p}$ is the reference viscoplastic displacement velocity, $a, b$ and $\alpha$ are empirical parameters. The term $Q_{c r}+a$ designates the limit resistance without the effect of time and the parameter $b$ quantifies the speed to reach the limit load. The viscous behavior of the soil is presented by the parameter $\alpha$ which presents the degree of influence of the rate of deformation. The load function is dependent on the speed of displacement capable of illustrating the time-dependent viscous behavior. This macroelement is tested under constant load for different application times in order to illustrate creep deformation of chalk.

\subsection{Results}

Firstly, a t-z calculation with a zero viscosity is performed to determine the static parameters, of the macroelement. A value of approximately zero is given to the parameter $\alpha$ to illustrate the static case in the absence of effects related to time. Table 1 summarizes the parameters of the elasto-viscoplastic model obtained by calibration of the curve load-head displacement obtained by this macroelement with that obtained by the t-z method with zero viscosity (figure 4.a).

Table 1. Static parameters of the macroelement

\begin{tabular}{|c|c|c|c|}
\hline $\mathbf{k}_{\mathbf{t}}(\mathbf{k N} / \mathbf{m})$ & $\mathbf{Q}_{\mathbf{c r}} \mathbf{( k N )}$ & $\mathbf{a}$ & $\mathbf{b}$ \\
\hline 170000 & 800 & 200 & 0.0018 \\
\hline
\end{tabular}

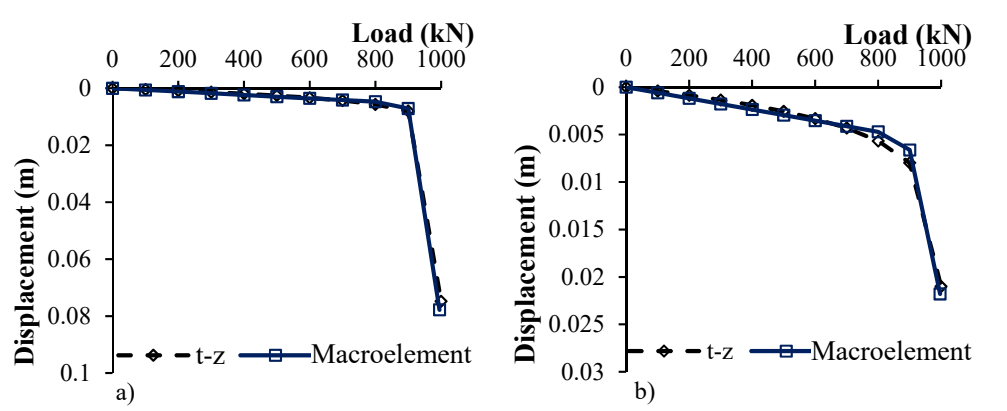

Fig. 4. Calibration of macroelment parameters from t-z calculation: a) Static parameters, b) viscous parameters $\eta=10000$ Pa.s, $\alpha=0.0012$

The same parameters were used for time dependent calculations using this macroelement in order to examine its ability to reproduce results observed by the t-z method. A t-z calculation was done, with $\eta=10000$ Pa.s. Calibration of the macroelement is done on the t-z model results in order to determine the viscous parameter $\alpha$ corresponding to the chosen value of $\eta$ (figure 4.b). The value of $\alpha$ found is 0.0012 .

Lastly, the effect of the load application duration is taken into account. Using the determined value of $\alpha$, a comparison between results obtained by the two methods with two different durations, 15 and $120 \mathrm{~min}$, is made (figure 5).
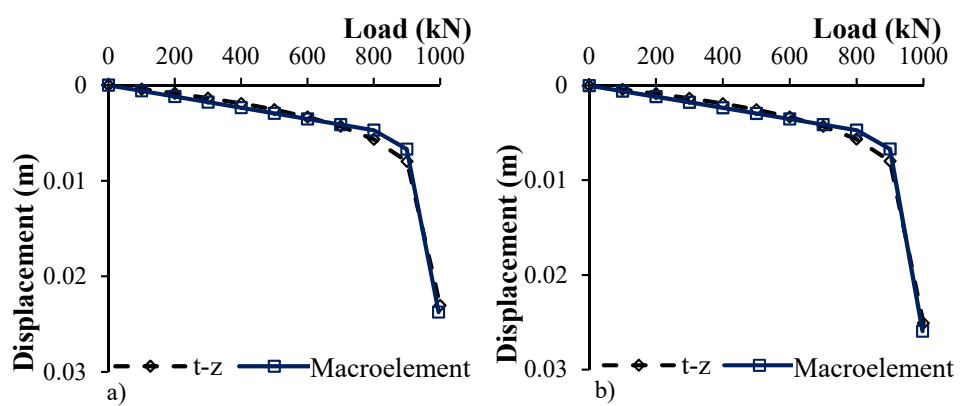

Fig. 5. Comparison of load-displacement curves at the top between the t-z and macroelement methods for a viscosity of $10,000 \mathrm{~Pa} . \mathrm{s}$ with different duration of load application: a) $15 \mathrm{~min}$, b) $120 \mathrm{~min}$ 
The results show a good consistency between these two methods even with the different durations of loading. The setting of the macroelement therefore does not depend on the duration of application of the load. The macroelement developed is capable to capture with reasonable reliability the viscous behavior of the chalk.

\section{Conclusion}

This paper focused on the study of behavior and dimensioning of piles in chalk under monotonic load. The chalk shows a noticeable creep under a constant load. In order to contribute to the prediction of the response of a pile under monotonic loading in chalk, a viscous model based on the transfer curves method is developed. This t-z curve method is used to model the friction along the pile shaft by an elasto-viscoplastic 1D rheological model derived from that of Bingham. A parametric study of this model showed the important influence of viscosity. In addition, a comparison of experimental results of pile load test and results from the developed model has shown a good consistency.

The results obtained with the macroelement are encouraging. Studies have shown that this method reduces computational costs, by reducing the calculation time, with a good agreement between t-z method and macroelement.

\section{References}

1. A. Dahou, PHD Thesis, University of Lille, (1995)

2. M. Datcheva, J. Theo. Appl. Mecha., 31, 53-64 (2001)

3. A. Monjoie, C. Schroeder, Third North Sea Chalk Symposium, (1990)

4. AFNOR, Calcul des fondations profondes - NF P 94 282, Norme française (2012)

5. API, Recommended practice for planning, designing and constructing fixed offshore platforms: working stress design. (2011)

6. H. Coyle, L. Reese, ASCE, 1-26 (1966)

7. M. Bustamante, O. Cambarieu, L. Gianeselli, Bull. Lab. Ponts et Chaussées (1980)

8. R. Nova, L. Montrasio, Géo, 41(2), 243-256(1991)

9. W. Wang, L. Sluys, R. De Borst, In. J. Num. Meth. Eng, 40, 3839-3864(1997)

10. L. Laloui, S. Leroueil, S. Chalindar, Cana. Geo., 1765-1777 (2008) 